\title{
The Oslo Opera House - Condition Analysis and Proposal for Cleaning, Protection and Maintenance of Exterior Marble
}

\author{
Pagona-Noni Maravelaki ${ }^{1(凶)}$, Lucia Toniolo ${ }^{2}$, Francesca Gheraldi ${ }^{2}$, \\ Chrysi Kapridaki ${ }^{1}$, and Ioannis Arabatzis $^{3}$ \\ 1 Technical University of Crete, 73100 Chania, Greece \\ pmaravelaki@isc.tuc.gr \\ 2 Politecnico di Milano, 20133 Milan, Italy \\ ${ }^{3}$ NanoPhos SA, Science and Technology Park of Lavrio, 19500 Lavrio, Greece
}

\begin{abstract}
The photo-oxidative degradation of the polymers applied in the past onto the marble slabs of the Oslo Opera House $(\mathrm{OOH})$ led to the yellowing of the coatings and/or to the formation of insoluble fractions of polymer. Despite the high reduction of the $b^{*}$ parameter (yellowness) observed after the mechanical abrasion, this cleaning treatment cannot be recommended for further application, due to the unacceptable removal of structural material during the abrasion. Cleaning of historic masonry has progressed significantly in recent years, thanks to methods that rely on nanoscience. Therefore, in this research project instead of using organic solvents exhibiting toxicity and poor performance, advanced nanostructured fluids, such as micelles solutions (MC) and microemulsions (ME), embedded in nanogels with industrial solvents were tested aiming at the efficient removal of the hydrophobic coatings from Carrara marble slabs. These nanogels are amphiphilic-based formulations using a system of water, oil and surfactant. However, lack of reproducibility characterized the application of these advanced nanogels, due to partly removal of the unevenly distributed polymer products. Therefore a three-step cleaning methodology was adopted including: the introduction of a chelating agent capable of dissolving the hard film created by the applied coatings, thus allowing any subsequent MC and ME solutions applied in the second step, to swell the polymeric chains and to facilitate their removal, which was further completed by suitable oxidants. Optical and scanning electron microscopy coupled with EDX, along with colorimetric measurements proved that the three-step cleaning tests applied to yellowed samples can successfully remove the colour discoloration.
\end{abstract}

Keywords: Oslo Opera House · Yellowing identification •

Cleaning with nanogels

\section{Introduction}

\subsection{Problem Identification}

The Norwegian National Opera and Ballet House (OOH) is a contemporary architecture designed by Snøhetta Architects and built in Oslo in the period 2000-2008 [1].

(C) Springer Nature Switzerland AG 2019

A. Moropoulou et al. (Eds.): TMM_CH 2018, CCIS 962, pp. 104-116, 2019.

https://doi.org/10.1007/978-3-030-12960-6_7 
The $\mathrm{OOH}$ was characterized by cladding mainly Carrara marble tiles and represents a connection of the city with the fjord landscape. The construction of the Opera House is the largest single culture-political initiative in contemporary Norway, and is therefore of outstanding importance for the country and its population.

In 2007-2008 the external marble surfaces of $\mathrm{OOH}$ underwent protective treatment aimed to facilitating subsequent regular cleaning operations. The treatment involved impregnation of all the surfaces with a fluorinated acryl copolymer, Faceal Oleo HD (PSS Interservice AG, Switzerland), and application of PSS 20 (PSS Interservice AG, Switzerland), a polysaccharide-based product for anti-graffiti protection of surfaces [2]. The functionality of Faceal Oleo HD for horizontal and vertical surfaces was guaranteed for 3 and 10 years, respectively, while the PSS 20 guaranteed for 3 years for all surfaces [2].

Despite cleaning operations that were regularly performed on the surfaces, a yellow colour discoloration of the horizontal surfaces appeared since 2007, inducing considerable aesthetic alteration to the aspect of the building.

Given the poor performance of Faceal Oleo HD [2], alternative products were tested in the course of 2011, after which the product showing the best performance was applied extensively in 2012 for a second impregnation. Official reports and documents of previous analytical investigations pointed out to the recovery of a high amount of silicon on surface, rather than the chemical nature of the relevant product.

Subsequent investigations aimed at clarifying the source and mechanism of the yellowing were also conducted but did not yield significant result. The early hypothesis that the fluorinated acryl polymer might be involved in the discolouration of marble through some degradation product was questioned on the grounds that the removal of the polymer was not able to eliminate the yellowing [2]. The new protective treatment did not prove to be effective in preventing the discolouration of marble, suggesting that the cause of the problem should be addressed differently.

To complicate things further, efforts made so far to remove the yellowing can hardly be considered satisfactory in the absence of an explanation and prevention of the phenomenon. Indeed, even though some cleaning methods have been proven able to bring the marble back to its pristine state [2], the high costs of the operation cannot be justified without gaining insights into the cause of discolouration and the strategy to effectively tackling the problem.

\subsection{Damage Classification and Actions to Be Undertaken After the Building Inspection}

In the framework of the Project "The Oslo Opera House - Condition analysis and proposal for cleaning, protection and maintenance of exterior marble" the research team of Politecnico di Milano and Technical University of Crete, inspected the $\mathrm{OOH}$ in 2015 and identified the sampling areas taking into account: (a) the different decay pattern morphology; (b) the different orientation and exposure of tiles to the weathering conditions; (c) the macroscopic differentiation in the color intensity of the observed yellowing; (d) the different surface finishing of the tiles (polished or rough); (e) the necessity to obtain the minimum but sufficient number of samples. 
In a first attempt to approach the condition survey according to the Standard Protocol EN 16096:2012 [3], we suggest that Symptoms are varying from CC0 (no symptoms) to CC2 (moderately strong symptoms), the Urgency risk classification ranges from UC0 (long term) to UC1 (intermediate term) and the possible measures range from RC1 (maintenance/preventive conservation) to RC2 (moderate repair and/or further investigation). Several characterization techniques were applied to investigate five marble samples of exposed tiles coming from $\mathrm{OOH}$. The key points of our investigation are:

- to gain a deep insight into the causes of the discolouration (chromatic alteration);

- to assess the conservation condition of Carrara marble, with particular regard to the severe environmental conditions;

- to set-up tailor-made cleaning methodology of the marble tiles (Fig 1).

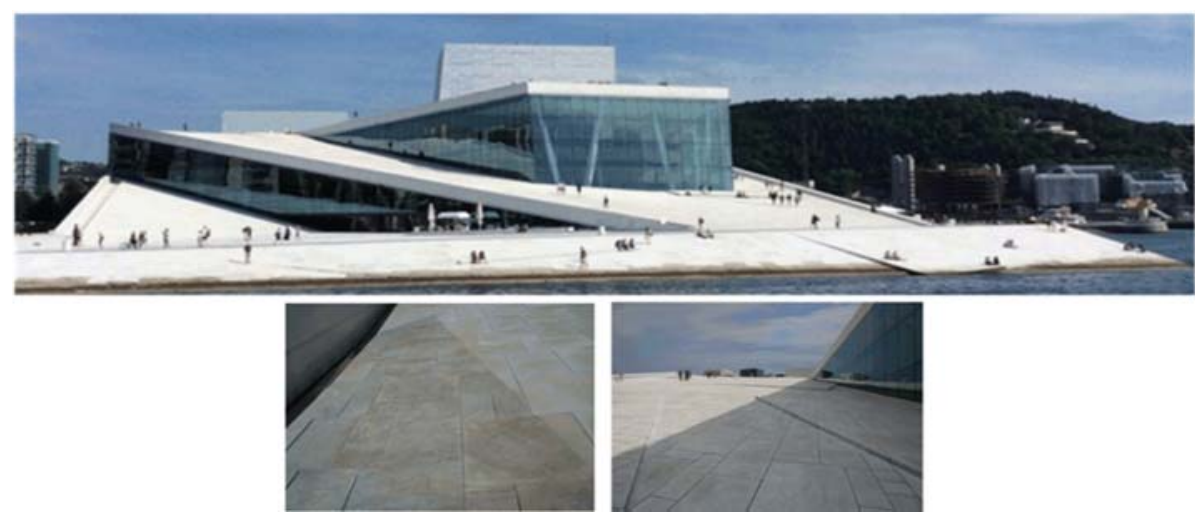

Fig. 1. Yellowing observed in the cladded façade with Carrara marble tiles of the Opera Oslo House.

\section{Materials and Methods}

\subsection{Marble Samples and Applied Coatings}

The Carrara marble tiles from $\mathrm{OOH}$ were demounted and collected from different areas of the building (P1, P2, P3A, P3B and P6), as reported in Fig. 2. In particular, from P1-P6 tiles several different small blocks were cut and sent to the laboratories in Italy and Greece.

The tiles coming from the building are characterized by different surface finishings: P1 tile has a "heavy bush hammered" finishing, P2 and P3B tiles have a "rough bush hammered" finishing and P3A and P6 tiles have a "sawn" finishing (Fig. 2) [2]. Some specimens, which were cut from the internal part of a Carrara marble tile "not exposed" (as referred from Statsbygg) on the roof of the building but conserved in the backyard at $\mathrm{OOH}$, were considered as reference sample for the unaltered material. Moreover, reference products of the two surface coatings used for the protection of the marble, 


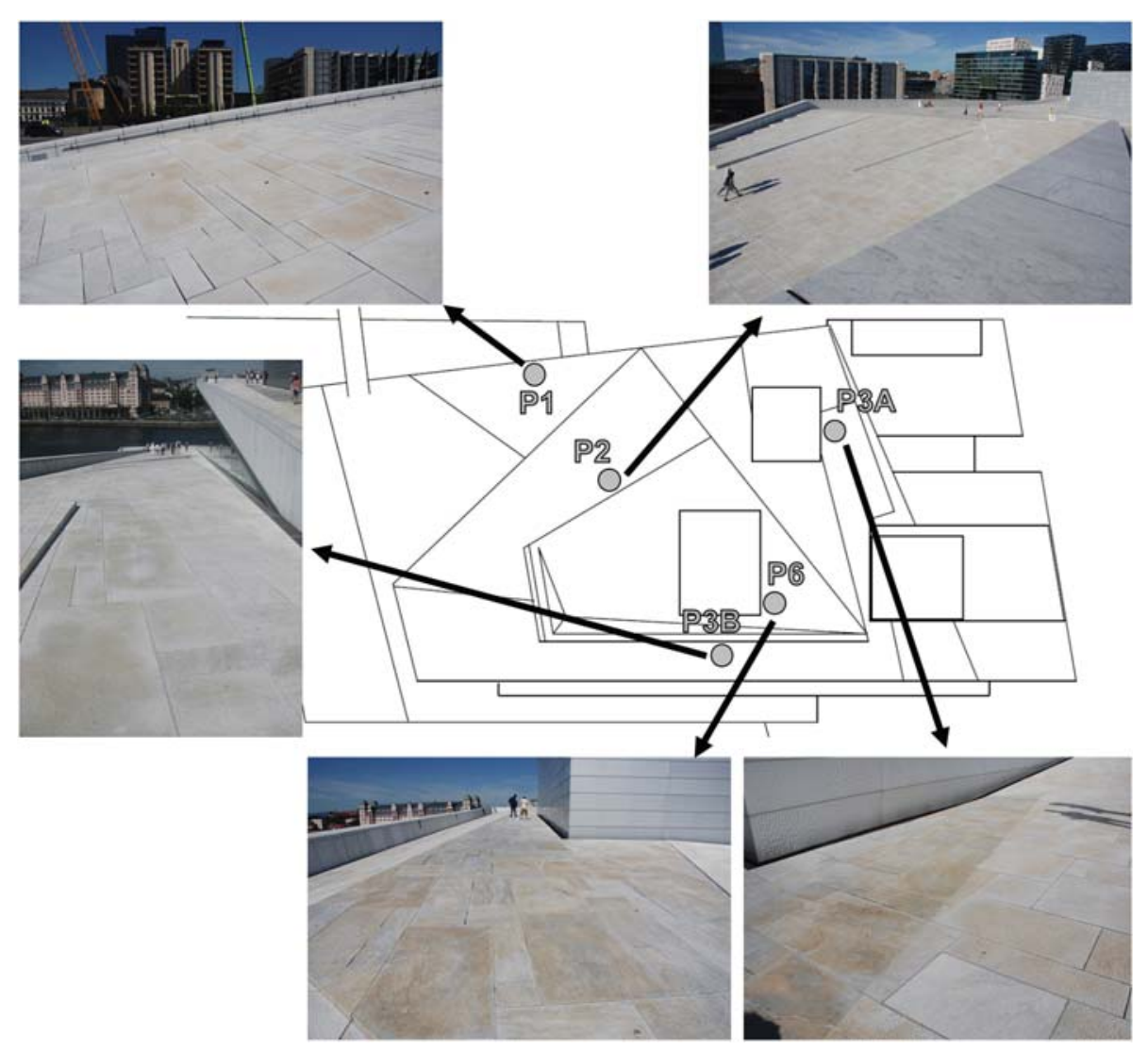

Fig. 2. Original localization of the marble tiles in the areas P1, P2, P3 and P6 of the building.

such as Faceal Oleo HD and PSS 20, PSS Interservice AG, Switzerland, were also investigated for comparison purposes.

\subsection{Instrumental Methods}

VIS spectrophotometric measurements were carried out on the surface of each tile with a Konica Minolta CM-600D instrument with a D65 illuminant at $8^{\circ}$, wavelength range between $360 \mathrm{~nm}$ and $740 \mathrm{~nm}$. Measurements were elaborated according to the CIE $\mathrm{L} * \mathrm{a} * \mathrm{~b} *$ standard colour system, which describes a colour with three different parameters, $\mathrm{L}^{*}, \mathrm{a}^{*}$, and $\mathrm{b}^{*}$, measuring respectively brightness, red/green and yellow/blue colour intensities. 180 measurements were performed on each area $(20 \times 20 \mathrm{~cm})$ of the exposed marble tiles, whereas 100 measurements were carried out on the samples taken from the unexposed marble tile. The average results of $L^{*} a^{*} b^{*}$ were used to calculate the colour difference $\Delta \mathrm{E}^{*}\left(\Delta \mathrm{E}^{*}=\left[\left(\mathrm{L}_{2}^{*}-\mathrm{L}_{1}^{*}\right)^{2}+\left(\mathrm{a}_{2}^{*}-\mathrm{a}_{1}^{*}\right)^{2}+\left(\mathrm{b}_{2}^{*}-\mathrm{b}_{1}^{*}\right)^{2}\right]^{1 / 2}\right)$ between the exposed and the unexposed marble tiles. 
From each tile, different micro-samples were collected from the surface. They were observed with a Leica M205C stereomicroscope equipped with a Leica DFC 290 video camera. Then, the morphology of the stone specimens was analyzed by Environmental Scanning Electron Microscopy (ESEM) and EDX analyses (Zeiss EVO 50 EP ESEM, equipped with an Oxford INCA 200 - Pentafet LZ4 spectrometer). The exposed surface, fresh fractured surface and the polished cross-section of the samples were investigated. Moreover, samples from the mortars collected from P2 and P6 marble tile were analyzed with ESEM-EDX.

In order to identify the protective treatments applied to the surface of both the unexposed and exposed marble tiles, a small amount of carbonatic powder was collected from the surface by a scalpel and the liquid extracts with appropriate solvents were deposited on $\mathrm{KBr}$ window then analyzed by Fourier-Transform Infrared Spectroscopy (FTIR), using a Nicolet 6700 spectrophotometer equipped with a DTGS detector (acquired between 4000 and $400 \mathrm{~cm}^{-1}$ with 128 acquisitions and $4 \mathrm{~cm}^{-1}$ resolution), after solvent evaporation. For comparison, the two surface coatings used for the protection of the marble tiles (Faceal Oleo HD and PSS 20) were analyzed by FTIR in the same conditions.

Moreover, from each tile, the deposits on the surface were collected with a pin and analyzed by micro-FTIR with Nicolet Continuum FTIR microscope equipped with a MCT detector (acquired between 4000 and $600 \mathrm{~cm}^{-1}$ with 128 acquisitions and $4 \mathrm{~cm}^{-1}$ resolution) using a micro compression diamond cell accessory.

\subsection{Cleaning Methodology}

To remove the previously applied treatments in $\mathrm{OOH}$ a cleaning methodology using appropriate pure solvents, nanofluids and nanogels in different cleaning supports, such as pulp poultices, cotton fabrics, gauze, agar was attempted. At the end of the tests, the poultices were removed and the surface was gently rinsed with hot water to remove possible residues. Table 1 lists the categories of the cleaning agents tested for each tile.

Table 1. Overview of the tailored-made cleaning formulations.

\begin{tabular}{l|l}
\hline Cleaning type & Cleaning code \\
\hline Pure and mixture of solvents & SLV \\
\hline Nanogels of advanced micelles solutions & NGMC \\
\hline Nanogels of microemulsions & NGEM \\
\hline Nanogels of micelles solutions and microemulsions & NGME \\
\hline Nanogels of microemulsions and micelles with Industrial solvents & NGEMS \\
\hline Oxidizing agents & OxA \\
\hline Chelating agents & CA \\
\hline
\end{tabular}

The nanogels and cleaning agents listed in Table 1 were specifically formulated for the cleaning of $\mathrm{OOH}$. These formulations consist of an anionic surfactant, water and solvents (a non-solvent and a good solvent for the polymer, respectively). The presence 
of the surfactant guarantees a decrease of the interfacial tension between the stone and the swollen polymer droplets and, therefore, the stabilization of these irregular structures. The shape of the water droplet test evidenced the polymer removal and the hydrophilic behaviour of the surface. Factors such as the application time of the cleaning agent and the ageing of the polymers influenced the removal of the applied materials. It is of great importance to have in mind that an aged polymer cannot be fully removed from the surface, probably because of the stone's porosity. Carrara marble is a low porosity stone but it is still a porous material and protective treatments penetrate inside the pores for a thickness of hundreds microns till $1 \mathrm{~mm}$. In particular, detachment and enlargement of crystalline grains' boundaries can be observed on the examined surface and this has surely increased the surface porosity of the marble tile, favouring the penetration of polymeric materials.

Cleaning tests, coupled with the direct observation of the morphological and aesthetical changes of the polymer film by means of optical and electronic microscopy and colorimetric measurements, have shown that the nanogels applied are not always efficient in polymer removal.

We suggest that this behavior, which is related to the superimposing of the different protective layers, made the system extremely difficult to be dissolved in a one-step advanced cleaning formulation. This was confirmed in lab experiments which were characterized by scarce reproducibility, due to the poor adsorption of the nanogel ingredients on the external layers of the polymeric coating. These structural differences can be responsible for the different cleaning efficacies and for the different kinetic profiles observed. However, the synergistic action between water, organic solvents and surfactant is of fundamental importance to obtain excellent cleaning performances. In fact, water causes the structural reorganization of the external polymeric layers, while the organic solvent swells the entire film increasing the mobility of the polymeric chains. At the same time, the surfactant interacts with the outer polymeric layers and decreases the interfacial energy at the marble/polymer interface, promoting the detachment of the polymer from the marble substrate.

At this point a smart selection on the key-steps recommended for cleaning was the introduction of a chelating agent capable of dissolving the PSS 20 polysaccharide layer, which created a hard film, not allowing any solvent to swell the polymeric chains. Therefore, after the sufficient removal of polysaccharide, as proven by the increase of water absorption, the nanogel application followed. The third key-step was the application of an oxidizing agent to facilitate the removal of polymeric residues decomposed from the previous treatments.

\section{Results and Discussion}

\subsection{Aesthetic, Morphological and Compositional Parameters of Yellowing}

Figure 3 shows the color parameters as obtained by colorimetric comparison between the yellowed and reference Carrara tiles. The color parameter $\mathrm{b}^{*}$, related to the 
yellowing, along with the total reflectance showed the highest diversification in the P6, P2 and P3B Carrara marble tiles comparing to the P1, P3A and reference marble.
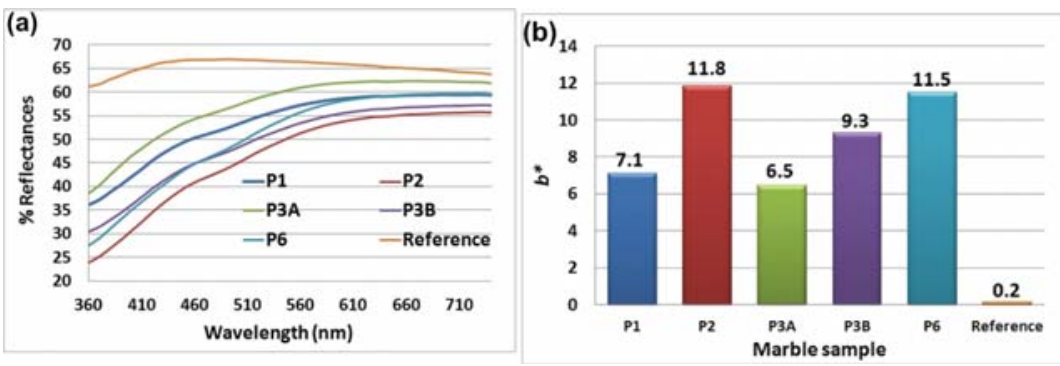

Fig. 3. (a) Reflectance spectra and (b) $b^{*}$ values of the yellowed marble originating from all the levels (P1, P2, P3A, P3B and P6) in comparison with the reference Carrara marble.

The samples collected from the surface of P1-P6 tiles are characterized by a significant inter- and intra-granular decohesion, where the crystals show corroded gemination planes and detached grains near the surface. This important degradation phenomenon of the crystal structure is well described also in the document "ICOMOSISCS: Illustrated glossary on stone deterioration patterns" [4]. It can be related to changes in temperature due to severe microclimatic condition of the building, with freezing-thawing cycles, to surface erosion phenomena caused by the weather precipitations (rain and snow) and to salt crystallization cycles caused by $\mathrm{NaCl}$ (halite) marine spray and therefore cyclic dissolution and re-crystallization inside the surface porosity of the stone. In Fig. 4, the electron microscopy images of the surface of sample collected from P6 tile are reported. This sample is characterized by an intense yellowing with $b^{*}=+11.5$, and an inter-granular decohesion. In particular, the surface of some grains is characterized by the presence of some "drops" of an organic material (Fig. 4, darker areas) with high signals of Si and $\mathrm{C}$, according to the elemental analysis, and ascribed to the presence of a siloxane treatment by means of solvent extraction and FTIR spectroscopy [5].

In addition to the solvent extractions, the deposits observed on the surface of the tiles were collected and analyzed by FTIR spectroscopy equipped with an optical microscope to analyze sample at the micron scale. The spectrum obtained from one of these micro-samples is reported in Fig. 5, and compared to the spectrum of the antigraffiti coating PSS 20 , based on vegetal polysaccharides. A significant overlay of the absorption bands of the two spectra can be observed, in particular, the absorption bands at 3349 (OH stretching), 2926 ( $\mathrm{CH}$ stretching), 1651 ( $\mathrm{OH}$ bending), $1566(\mathrm{C}=\mathrm{O}$ stretching in COO-), 1410 and 1365 (CH bending) and the main peak at $1012 \mathrm{~cm}^{-1}$ related to $\mathrm{C}-\mathrm{C}$ and $\mathrm{C}-\mathrm{O}$ stretching of the pyranose ring of polysaccharides [6]. This result clearly indicates that these yellow-brown deposits are residuals of PSS 20 treatment. 

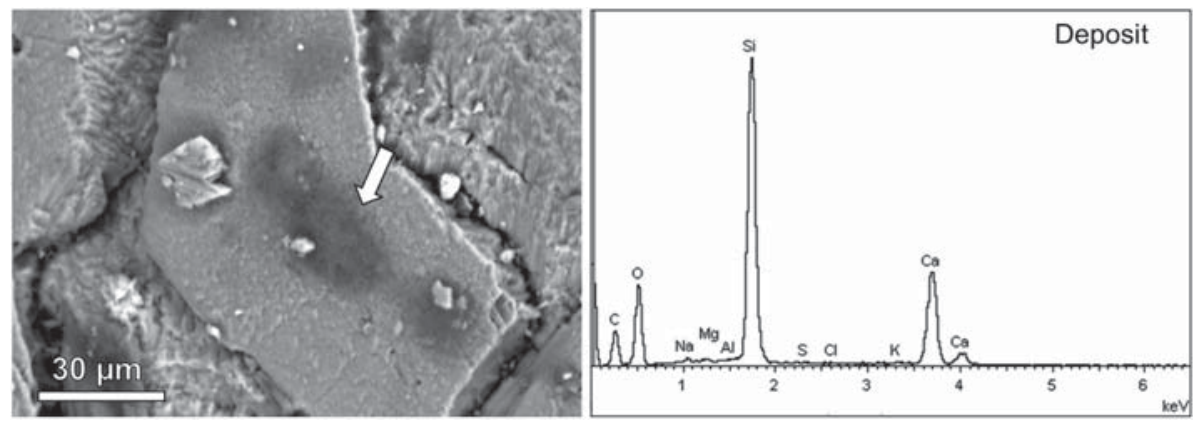

Fig. 4. Electron microscopic image of a crystal of P6 sample with accumulation of an organic treatment and the corresponding EDX spectrum.

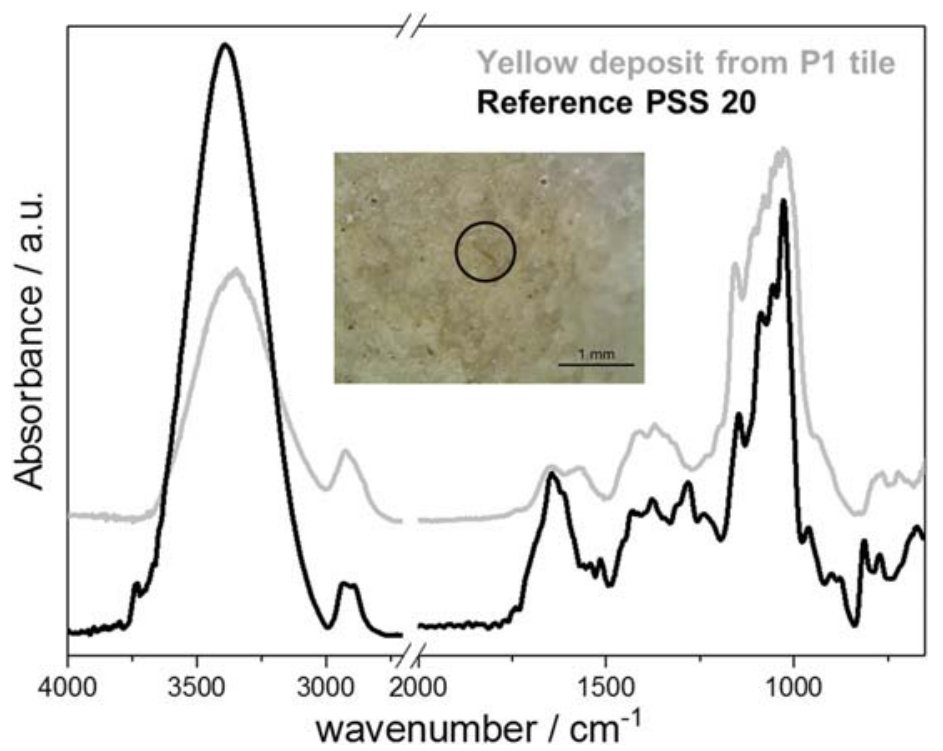

Fig. 5. Microscopic image of yellow-brown surface deposit on P1 tile with the FTIR spectrum of the deposit (grey) and the spectrum of the vegetable polysaccharide-based treatment (PSS 20) (black). (Color figure online)

\subsection{Cleaning Results}

Research has been carried out in order to remove the polymeric Faceal Oleo HD and PSS 20 that were used as protective and antigraffiti, respectively, and have been recognized as responsible for the discoloration due to their photochemical instability. Our research studies on cleaning were mainly focused on marbles originating from P2 and P6 levels which exhibit the most intense staining comparing to the other levels.

The most commonly used method to remove deteriorated polymers from treated surfaces is the use of solvents usually organic, such as acetone, xylene, toluene, ethyl 
acetate, alcohols or mixtures of them. Unfortunately, these solvents are not only toxic and harmful to workers' health, but they might induce further migration of the dissolved polymer inside the stone. Moreover, when solvents are used for the removal of the applied polymer, swelling of the polymer can be observed on the surfaces, thus demanding further mechanical abrasion in order the polymer removal to be completed. However, through this abrasion, especially in porous materials, parts of the material underneath could be disrupted. As expected, none of the colour parameters were improved and in almost all cases the spreading of the polymers inside the bulk of the marble resulted in a decrease of lightness and an increase of the yellowing parameter $\mathrm{b}^{*}$, as illustrated in Fig. 6.

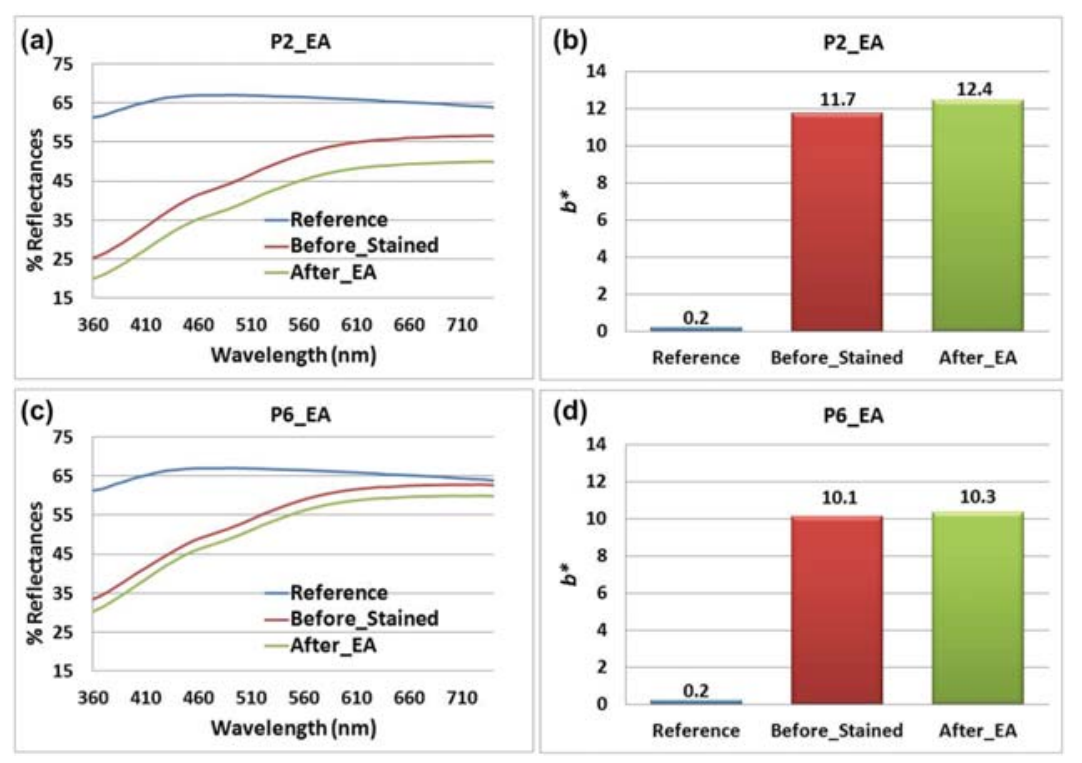

Fig. 6. (a) and (c) Reflectance spectra of the surfaces and (b) and (d) b* parameter values of P2 and P6 marbles before and after the immersion in ethyl acetate.

The core of our research was to assess the efficiency of advanced cleaning systems, such as nanogels with microemulsions and/or micellar solutions [7, 8]. Therefore, the performance of the designed solutions applied with a nanogel support was also assessed in all of the samples. However, the changes induced from the applied treatments in the colour parameters and the reflectance spectra indicated insignificant improvement of the colour parameters with lack of reproducibility.

The previously described attempts to remove the yellowing resulted in a poor performance due to durability of the applied coatings and the superimposing of different layers rendering their detachment extremely difficult. It was deemed important to combine the potentiality and efficiency of advanced formulations recently proposed, such as microemulsions and micellar solutions with well-established organic industrial solvents (NGEMS). Following this direction several promising results were obtained 
and the relevant formulations were designed in close collaboration with NanoPhos. However, no reproducibility characterized the application of these advanced cleaning systems and in some cases the state of the treated surface was clearly worsened (Fig. 7b).
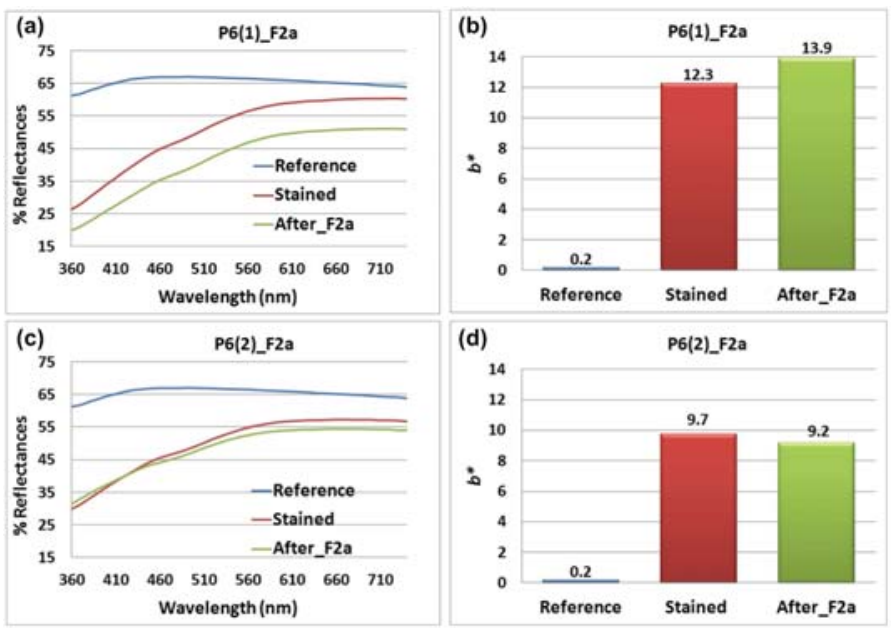

Fig. 7. P6 marble tile treated with NGEMS (F2a agent): (a and c) Reflectance spectra of the surfaces and (b and d) $b^{*}$ parameter values before and after cleaning.

The inefficiency of the advanced nanogel system NGEMS reinforced with the most commonly used industrial solvents into removing the yellowing, addressed research activities into adopting a scenario with a separate key-step before advancing with the application of the nanogels. This determinant cleaning key-step comprised of an application of a chelating agent capable of dissolving the polysaccharide film and capturing the $\mathrm{Ca}^{+2}$ ions in order to accomplish the polymeric film detachment [9]. Then the advanced nanogel system was applied and the coating removal was accomplished by a final application of formulations with oxidants.

At this point no further information could be provided about the chemical composition and specific ingredients of the three-step cleaning methodology due to constraints with the industrial partner and the relative patent application.

Table 2 lists the changes induced after the three-step cleaning in the $b^{*}$ colour parameter and the total colour difference $\Delta \mathrm{E}$. The three - step cleaning methodology applied to $\mathrm{P} 1-\mathrm{P} 3 \mathrm{~B}$ marble tiles induced more than $50 \%$ change in the $b^{*}$ parameter related to yellowness, while luminosity increased too. The P6 tile exhibited a $42 \%$ reduction of the yellowness and the highest total colour difference towards yellowed and reference sample. No morphological changes were observed after the three-step cleaning, as illustrated in the electron microscopy image of P6 tile in Fig. 8 comparing 
Table 2. Change of colorimetric parameters after cleaning

\begin{tabular}{l|l|l|l}
\hline Samples & \% Change of $\mathrm{b}^{* \mathrm{a}}$ & $\Delta \mathrm{E}^{* \text { a(yellowed) }}$ & $\Delta \mathrm{E}^{* \mathrm{~b} \text { (reference) }}$ \\
\hline P1 & -53.3 & 4.7 & 3.6 \\
\hline P2 & -54.3 & 7.5 & 7.4 \\
\hline P3A & -64.9 & 3.9 & 2.7 \\
\hline P3B & -54.9 & 5.3 & 5.6 \\
\hline P6 & -42.4 & 8.0 & 8.8
\end{tabular}

${ }^{\mathrm{a}}$ referred to yellowed, ${ }^{\mathrm{b}}$ referred to Reference

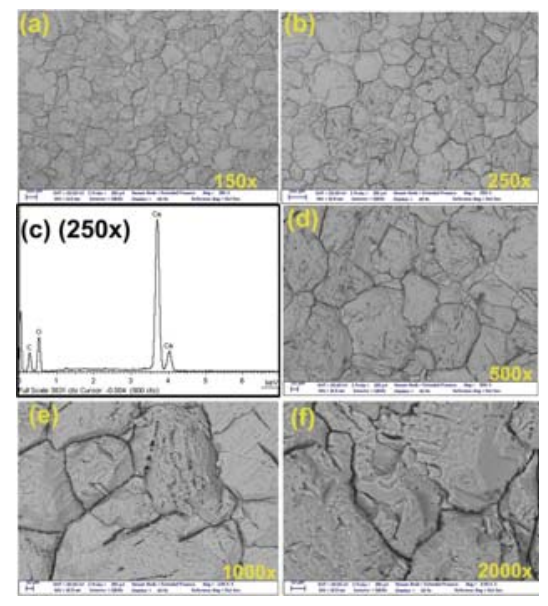

Fig. 8. SEM-EDX images of the cleaned P6 at different magnifications: (a) 150x, (b) 250x, (c) EDX spectrum (250x), (d) 500x, (e) 1000x and (f) 2000x.

with the corresponding image of the yellowed sample in Fig. 4, and in the macroscopic images of Fig. 9 for the P3B. The cleaned P6 surface exhibited a grain detachment and etching along with a visible boundaries decohesion, but not to a greater extent than that observed for the yellowed counterparts.

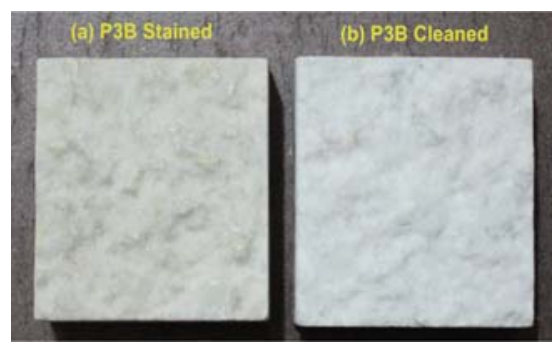

Fig. 9. Macroscopic images of the yellowed and cleaned surface for the P3B tile. (Color figure online) 


\section{Conclusions}

The photo-oxidative degradation of the polymers applied onto $\mathrm{OOH}$ marble tiles, such as Faceal Oleo and PSS20 led to the yellowing of the coatings and to the formation of insoluble fractions of polymer, which makes the treatment not compatible and impossible to be completely removed. The removal of these polymeric coatings was therefore one of the main challenges in this scientific project, without suggesting mechanical abrasion treatment often associated with unacceptable removal of structural material.

Advanced cleaning strategies using nanogels with microemulsions and micelles exhibited low performance and lack of reproducibility, due to the partial removal of the polymers. A smart selection on the key-steps recommended for cleaning was the introduction of a chelating agent capable of dissolving the hard coating not allowing any solvent to swell the polymeric chains. The polysaccharide and the silicon based layers removal was checked and proved by the absence of Si in the electron microscopy study. Then, as a second key-step the nanogel application followed. The third key-step was the application of an oxidizing agent assisting in the removal of polymeric residues decomposed from the previous treatments.

Optical and scanning electron microscopy coupled with EDX and colorimetric measurements proved that the three-step cleaning tests applied to yellowed samples can successfully remove the residues of the polymer degradation and maintain the surface aesthetical characteristics.

Acknowledgements. This research was carried out in the framework of the competitive Project "The Oslo Opera House - Condition analysis and proposal for cleaning, protection and maintenance of exterior marble" funded by Statsbygg, Oslo (Norway). The Authors gratefully thank Dr. Adriana Eidsvik and all the Statsbygg staff for the constant support and advice during the implementation of the Project.

\section{References}

1. http://snohetta.com/projects

2. Nilsen, B.: Maintenance operations management (MOM) information for the project: the oslo opera house - condition analysis and proposal for cleaning, protection and maintenance of exterior marble. s.l.: Betong Consult as, 04.11.15. doc 201501489 Operaen i Oslo - Utendørs marmor, rev. 02

3. 1: Standard Protocol EN 16096:2012, Conservation of cultural property - Condition survey and report of built cultural heritage

4. ICOMOS-ISCS: Illustrated glossary on stone deterioration patterns, ICOMOS. International Scientific Committee for Stone, Paris (2008)

5. Gherardi, F., Kapridaki, C., Roveri, M., Gulotta, D., Maravelaki, P.N., Toniolo, L.: The deterioration of Apuan white marble in contemporary architectural context. J. Cult. Herit. (2018, in press)

6. Oh, S.Y., Yoo, D.I., Shin, Y., Seo, G.: FTIR analysis of cellulose treated with sodium hydroxide and carbon dioxide. Carbohydr. Res. 340, 417-428 (2005) 
7. Baglioni, P., et al.: Micelle, microemulsions, and gels for the conservation of cultural heritage. Adv. Colloid Interface Sci. 205, 361-371 (2014)

8. Grassi, S., Favaro, M., Tomasin, P., Dei, L.: Nanocontainer aqueous systems for removing polymeric materials from marble surfaces: a new and promising tool in cultural heritage conservation. J. Cult. Herit. 10(3), 347-355 (2009)

9. Deng, J., Shi, Z., Li, X., Liu, H.: Soluble Polysaccharides Isolation and Characterization from Rabbiteye Blueberry (Vaccinium ashei) Fruits. BioResources 8(1), 405-419 (2013) 